\title{
CXCR3A contributes to the invasion and metastasis of gastric cancer cells
}

\author{
CHENGGANG YANG, WANLEI ZHENG and WENFENG DU \\ Department of Gastrointestinal Surgery, Liaocheng People's Hospital, Liaocheng, Shandong 252000, P.R. China
}

Received March 1, 2016; Accepted July 7, 2016

DOI: $10.3892 /$ or.2016.4953

\begin{abstract}
CXCR3, belonging to CXC chemokine receptors, has been identified to be overexpressed in various kinds of tumors. There are three mRNA variants of CXCR3 (CXCR3A, CXCR3B and CXCR3alt) in human cells. The functions of major CXCR3 isoforms (CXCR3A, CXCR3B) have been reported in some tumors including prostate and breast cancer. However, the effects of CXCR3A and CXCR3B on gastric cancer cell progression remain unknown. The present investigation found that CXCR3A mRNA level was upregulated but CXCR3B mRNA level was downregulated in gastric cancer cells and tissues. In vitro growth analysis showed that CXCR3A acted as a positive mediator in regulating cell growth, whereas CXCR3B exerted the opposite effect. In vitro invasion and migration assays showed that CXCL10 promoted gastric cancer cell invasion and migration via CXCR3A, but not CXCR3B. Moreover, knockdown of CXCR3A inhibited cell growth and metastasis in vivo. Additionally, CXCR3A knockdown attenuated matrix metalloproteinase (MMP)-13 and IL- 6 expression, and reduced ERK1/2 activation. Together, these data suggest that CXCR3A contributes to the growth, invasion and metastasis of gastric cancer cells in vitro and in vivo, and thus may be a key mediator of gastric cancer progression.
\end{abstract}

\section{Introduction}

Gastric cancer is one of the most common aerodigestive tract malignancies and is the third leading cause of cancer-related deaths in the world (1). Although therapy for cancer has been greatly improved, the prognosis of advanced gastric cancer is still poor because of tumor invasion and metastasis (2). Thus, uncovering the molecular mechanisms of gastric cancer to

Correspondence to: Dr Chenggang Yang, Department of Gastrointestinal Surgery, Liaocheng People's Hospital, 67 Dongchang West Road, Dongchangfu District, Liaocheng, Shandong 252000, P.R. China

E-mail: yangcggang@yeah.net

Key words: CXCR3A, CXCL10, invasion, growth, metastasis seek new molecular markers for early detection of gastric cancer is necessary.

As a member of $\mathrm{G}$ protein-coupled receptor family, $\mathrm{CXCR} 3$ binds to ELR-negative CXC chemokines such as CXCL9, CXCL10, CXCL11 and CXCL4, and participates in various human diseases including chronic inflammation (3), immune dysfunction (4) and cancer (5). CXCR3 has been reported to be upregulated in many cancers including colon cancer and basal cell carcinomas, and is closely associated with tumorigenesis and prognosis $(6,7)$. Three variants of CXCR3 (CXCR3A, CXCR3B and CXCR3alt) have been identified in human cells. Many studies have focused on the role of the major CXCR3 isoforms (CXCR3A and CXCR3B) in cancer progression, and have found that these variants of CXCR3 may exert different, even opposite, functions in cancer (8). It has been reported that CXCR3A is upregulated in clear cell ovarian cancer, but CXCR3B is downregulated (9). Studies also found that CXCR3A mRNA level is upregulated while CXCR3B mRNA is downregulated in prostate cancer specimens, and downregulation of CXCR3A but upregulation of CXCR3B significantly inhibits prostate cancer cell proliferation and invasion $(10,11)$. In gastric cancer, a recent study showed that upregulation of CXCR3B correlates with favorable prognosis of gastric cancer patients (12). However, the role of CXCR3A in gastric cancer remains unclear.

In this study, we examined the expression of CXCR3 variants in gastric cancer tissues and cells, and tried to uncover the functions of CXCR3A and CXCR3B in gastric cancer cell invasion, growth and metastasis in vitro and in vivo.

\section{Materials and methods}

Cell culture and reagents. All cell lines were purchased from Cell Resource Center of the Chinese Academy of Medical Science (Beijing, China). Gastric epithelium immortalized GES-1 cells were cultured in DMEM medium supplemented with $10 \%$ fetal bovine serum (FBS), while gastric cancer MKN28, SGC-7901 and AGS cells were cultured in RPMI-1640 medium supplemented with 10\% FBS. Cells were incubated in a humidified incubator $\left(37^{\circ} \mathrm{C}, 5 \% \mathrm{CO}_{2}\right)$. CXCL10 was obtained from Sigma-Aldrich (St. Louis, MO, USA).

Antibodies against CXCR3, ERK1/2 and $\beta$-actin were obtained from Santa Cruz Biotechnology (Santa Cruz, CA, USA). Antibody against phosho-ERK1/2 was obtained from Cell Signaling Technology (Danvers, MA, USA). 
Tumor tissues. Gastric cancer tissues and their corresponding non-cancerous tissues were obtained from the Department of Pathology $(n=40)$, Liaocheng People's Hospital. All specimens were approved by the Committee for Ethical Review of Research in the hospital, and histopathologically confirmed by the pathologist.

Real-time PCR. Total RNA from the specimens and cancer cell lines were extracted using TRIzol reagent (Invitrogen, Carlsbad, CA, USA), according to the manufacturer's instructions. Reverse transcription PCR was performed with total RNA and First Strand cDNA Synthesis kit (Fermentas, Glen Burnie, MD, USA). Then the cDNA was subjected to real-time PCR with ABI Prism 7700 Sequence Detection System (Applied Biosystems, Foster City, CA, USA). Primers used in the real-time PCR are as follows: CXCR3A forward, 5'-ACCCAGCAGCCAGAGCACC-3' and reverse, 5'-TCA TAGGAAGAGCTGAAGTTCTCCA-3'; CXCR3B forward, 5'-TGCCAGGCCTTTACACAGC-3' and reverse, 5'-TCGGCG TCATTTAGCACTTG-3'; CXCR3alt forward, 5'-CCAATA CAACTTCCCACAGGGGT-3' and reverse, 5'-GTCTCAGAC CAGGATGAATCCCG-3'; $\beta$-actin forward, 5'-CATGTACGT TGCTATCCAGGC-3' and reverse, 5'-CTCCTTAATGTC ACGCACG-3'. $\beta$-actin expression was used as an internal control. The results were assessed using the $2^{-\Delta \Delta \mathrm{CT}}$ method.

Western blot analysis. After washing with cold PBS, cells were lysed in RIPA lysis buffer. Total protein concentration was determined using BCA Protein Assay kit (Pierce Biotechnology, Inc., Rockford, IL, USA). A total of $40 \mu \mathrm{g}$ protein was resolved by SDS-PAGE and then transferred to PVDF membrane. The membrane was blocked in 5\% BSA for $1 \mathrm{~h}$, and incubated with primary antibodies at $4^{\circ} \mathrm{C}$ overnight. Next, the membrane was incubated with appropriate secondary antibodies and developed by enhanced chemiluminescence (ECL) Plus detection system (Pierce Biotechnology, Inc.).

siRNA and shRNA. CXCR3A siRNA (siCXCR3A) and CXCR3B siRNA (siCXCR3B) were purchased from Genchem Biotechnology Co. (Shanghai, China) for transient silence CXCR3A and CXCR3B expression, respectively. A scramble siRNA was purchased as control siRNA (siNC). CXCR3A shRNA (shCXCR3A) was also purchased from Genchem Biotechnology Co. to stably silence CXCR3A expression and a scramble shRNA was used as control shRNA (shNC). Cells were transfected with siRNA or shRNA using Lipofectamine 2000 (Invitrogen). Stable shRNA clone was selected by G418. The knockdown efficiency was determined by real-time PCR.

In vitro invasion and migration assays. In vitro invasion and migration assays were performed with 24-well Transwell plates (Corning Incorporated, Corning, NY, USA). The upper filters were coated with $50 \mu$ l Matrigel for invasion assay, whereas without Matrigel for migration assay. Cells were resuspended in RPMI-1640 medium at a density of $5 \times 10^{5}$ cells $/ \mathrm{ml}$. A total of $200 \mu \mathrm{l}$ of cell suspension was added into the upper chambers while $500 \mu \mathrm{l}$ of RPMI-1640 medium containing 20\% FBS was added into the lower chambers. After $12 \mathrm{~h}$, the cells that passed through the membranes were fixed with methanol and stained with crystal blue. Cell number was counted in seven random fields under a light microscope.

In vitro cell counting assay. Cells were seeded into a 24-well plate at a density of $1 \times 10^{4}$ cells/well. Further, cells were stimulated with or without CXCL10 and then incubated in the medium for the indicated time. Next, the cells were trypsinized, stained with trypan blue and counted in a hemocytometer.

$C C K-8$ assay. Cells were seeded in a 96-well plate at a concentration of $1 \times 10^{3}$ cells/well. Further, cells were stimulated with or without CXCL10 and then incubated in the medium for the indicated time. Next, CCK- 8 was added into the plate and incubated for $2 \mathrm{~h}$. Optical density (OD) was measured by microplate reader (Bio-Rad Model 680) at $490 \mathrm{~nm}$.

ELISA assay. After the cell supernatant or tumor tissues in the mice were collected, matrix metalloproteinase (MMP)-13 and IL-6 ELISA kits (Invitrogen) were used to measure the protein level of MMP-13 and IL-6, respectively, according to the manufacturer's instructions.

In vivo growth and metastasis assays. The male BABL/c nude mice (4-week old) were maintained in the pathogen-free conditions. All procedures were conducted following the Animal Care and Use Committee guidelines of Liaocheng People's Hospital. Cells at a density of $3 \times 10^{5}$ cells $/ 100 \mu 1$ were subcutaneously injected in the back of the mice $(n=8)$. The tumor formed in $\sim 3$ days. Then the length (L) and width (W) of tumors in mice were measured every week. Tumor volume was calculated with the formula of $\left(\mathrm{Lx} \mathrm{W}^{2}\right) / 2$. Eight weeks later, the mice were sacrificed and tumors were lysed to detect MMP-13 and IL- 6 expression and ERK1/2 activation. The livers of all mice were fixed in $4 \%$ paraformaldehyde and sectioned into slices. Each slice was stained with haematoxylin and eosin (H\&E) and then the number of micrometastasis in the livers was observed and counted under a light microscope.

Statistical analysis. Experiments were performed at least three times. Statistical analysis was performed using SPSS 17.0 (SPSS, Inc., Chicago, IL, USA). Student's t-test was used for comparison between two groups whereas one-way analysis of variance (ANOVA) was used for comparison among multiple groups. $\mathrm{P}<0.05$ was considered as statistically significant.

\section{Results}

CXCR3A is overexpressed in gastric cancer cells and tissues. By western blot analysis, we found that CXCR3 was overexpressed in gastric cancer MKN28, SGC-7901 and AGS cells as compared to gastric epithelium immortalized GES-1 cells (Fig. 1A). The mRNA expression of its three variants in gastric cancer cells were further assessed by real-time PCR. The results showed that CXCR3A expression was upregulated but CXCR3B expression was downregulated in gastric cancer cells. CXCR3alt mRNA expression showed no significant change in any of the detected gastric cancer cells compared with GES-1 cells (Fig. 1B). In addition, CXCR3A expression was increased but CXCR3B expression was decreased 
A

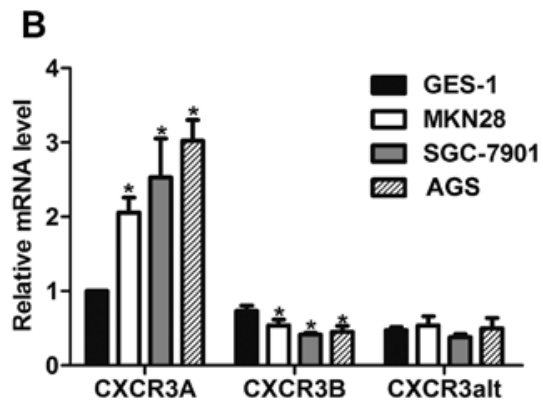

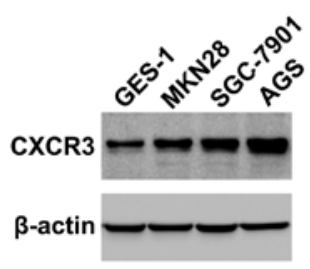

C
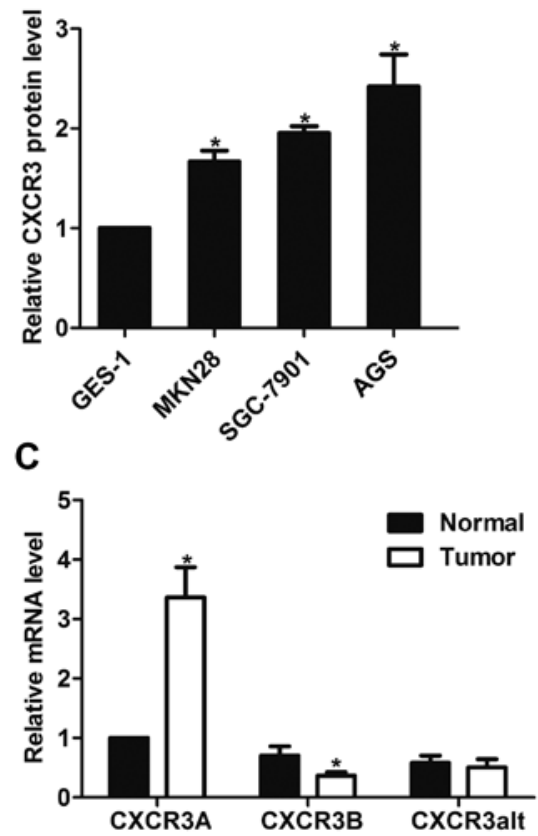

Figure 1. Expression of CXCR3A, CXCR3B and CXCR3alt in gastric cancer cells and tissues. (A) The protein expression of CXCR3 and (B) the mRNA expression of CXCR3A, CXCR3B and CXCR3alt, in GES-1, MKN28, SGC-7901 and AGS cells. (C) The mRNA expression of CXCR3A, CXCR3B and CXCR3alt in tumor tissues and the corresponding gastric tissues (normal). ${ }^{*} \mathrm{P}<0.05$.

A

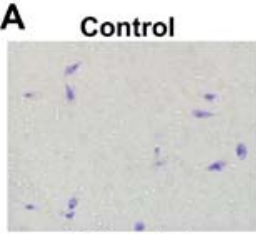

$100 \mathrm{ng} / \mathrm{ml}$

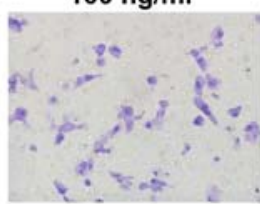

B

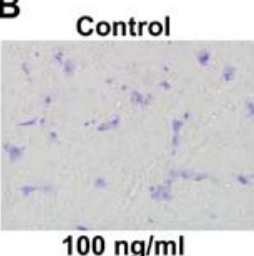

$100 \mathrm{ng} / \mathrm{ml}$

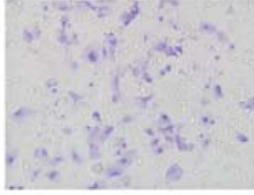

$50 \mathrm{ng} / \mathrm{ml}$

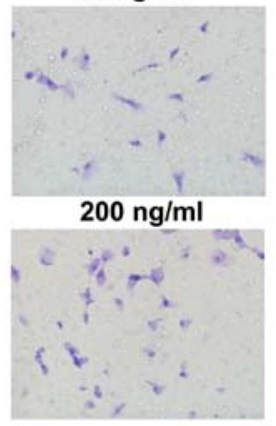

$50 \mathrm{ng} / \mathrm{ml}$

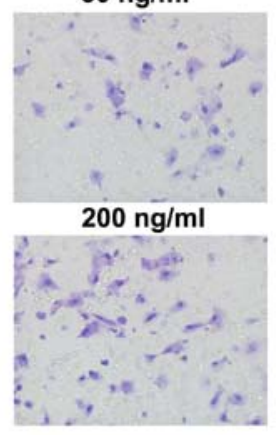

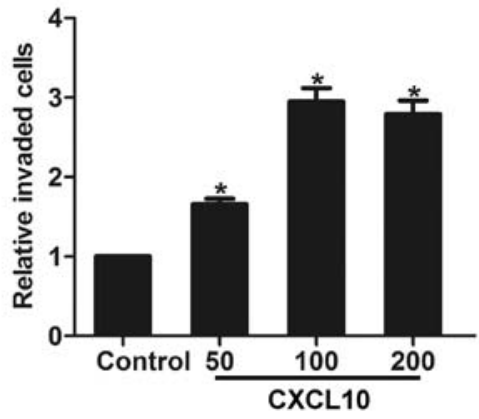

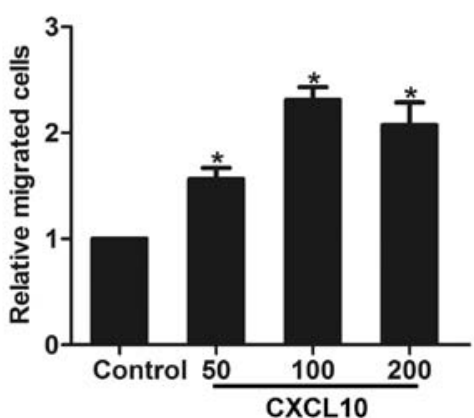

Figure 2. Effects of CXCL10 on cell invasion and migration of AGS cells. AGS cells were stimulated with 50, 100 and $200 \mathrm{ng} / \mathrm{ml} \mathrm{CXCL10,} \mathrm{respectively,} \mathrm{for}$ $12 \mathrm{~h}$. (A) In vitro invasion and (B) migration assays were performed using Transwell plates. " $\mathrm{P}<0.05$.

in gastric cancer tissues as compared to the corresponding gastric tissues, whereas no significant change was observed for CXCR3alt expression (Fig. 1C).

Activation of CXCR3 by CXCL10 promotes the invasion and migration of gastric cancer cells in vitro. To investigate the role of CXCR3 in gastric cancer cell invasion and migration in vitro, we stimulated AGS cells with different concentrations of CXCL10 (CXCR3 ligand) to activate CXCR3. Invasion and migration assays showed that CXCL10 promoted the invasion and migration of gastric cancer cells, in a dose-dependent manner (Fig. 2A and B), indicating that activation of CXCR3 
may be involved in gastric cancer cell invasion and migration in vitro.

Knockdown of CXCR3A inhibits gastric cancer cell invasion and migration in vitro. We then focused on the role of CXCR3A and CXCR3B in gastric cancer cells, as they both showed significant changes in gastric cancer tissues and cells. After confirming the specific knockdown efficiency of CXCR3A and CXCR3B in AGS cells (Fig. 3A), the effects of CXCR3A and CXCR3B on cell invasion and migration were detected by in vitro invasion and migration assay. We found that CXCL10 (100 ng/ml) stimulated the invasion and migration in siNC cells. In contrast, knockdown of CXCR3A inhibited CXCL10-induced cell invasion and migration, whereas knockdown of CXCR3B had little effect on the invasion and migration (Fig. 3B and C). These results confirm that it is CXCR3A, but not CXCR3B, that participates in gastric cancer cell invasion and migration in vitro.

CXCR3A regulates $M M P-13$ and $I L-6$ secretion and ERK1/2 activation in vitro. We then found that MMP-13 and IL-6 secretion was increased in siNC cells after CXCL10 stimulation for $12 \mathrm{~h}$. However, knockdown of CXCR3A greatly suppressed the CXCL10-induced MMP-13 and IL-6 secretion (Fig. 4A and B). Moreover, after stimulated with CXCL10 for $30 \mathrm{~min}$, ERK1/2 kinases were activated in siNC cells. In contrast, the CXCL10-mediated ERK1/2 activation was markedly attenuated in siCXCR3A cells (Fig. 4C).

CXCR3A promotes the growth of gastric cancer in vitro. Next, we found that CXCL10 treatment dose-dependently promoted the growth of AGS cells (Fig. 5A and B). To test the role of CXCR3A and CXCR3B in the growth of gastric cancer cells in vitro, cells were transfected with siNC, siCXCR3A and siCXCR3B, respectively, and then stimulated with $100 \mathrm{ng} / \mathrm{ml}$ CXCL10 for $72 \mathrm{~h}$. The results showed that knockdown of CXCR3A inhibited the CXCL10-mediated growth of AGS cells, whereas knockdown of CXCR3B promoted cell growth in vitro (Fig. $5 \mathrm{C}$ and $\mathrm{D})$.

CXCR3A is involved in the growth and metastasis of gastric cancer cells in vivo. Further, an AGS cell clone that stably silenced the expression of CXCR3A was established and confirmed by real-time PCR (Fig. 6A). Then the shCXCR3A and shNC cells were injected subcutaneously at the back of the mice, respectively. The tumor volume was assessed through measuring the length and width of the tumor in mice each week. The results showed that the tumor size in shCXCR3A group was much smaller than that in shNC group (Fig. 6B). Eight weeks later, the mice were sacrificed, micrometastasis in the liver section was counted under a microscope (Fig. 6C). We found that liver metastasis was observed in all eight mice in the shNC group, whereas it was observed in six mice in the shCXCR3A group. We counted the number of liver micrometastasis in all $\mathrm{H} \& \mathrm{E}$ slices, and found that knockdown of CXCR3A decreased the number of micrometastasis in the liver (Fig. 6D). In addition, we found that the expression of MMP-13 and IL-6 in tumor tissues of shCXCR3A group were significantly decreased (Fig. 7A and B), and the activation of ERK1/2 was
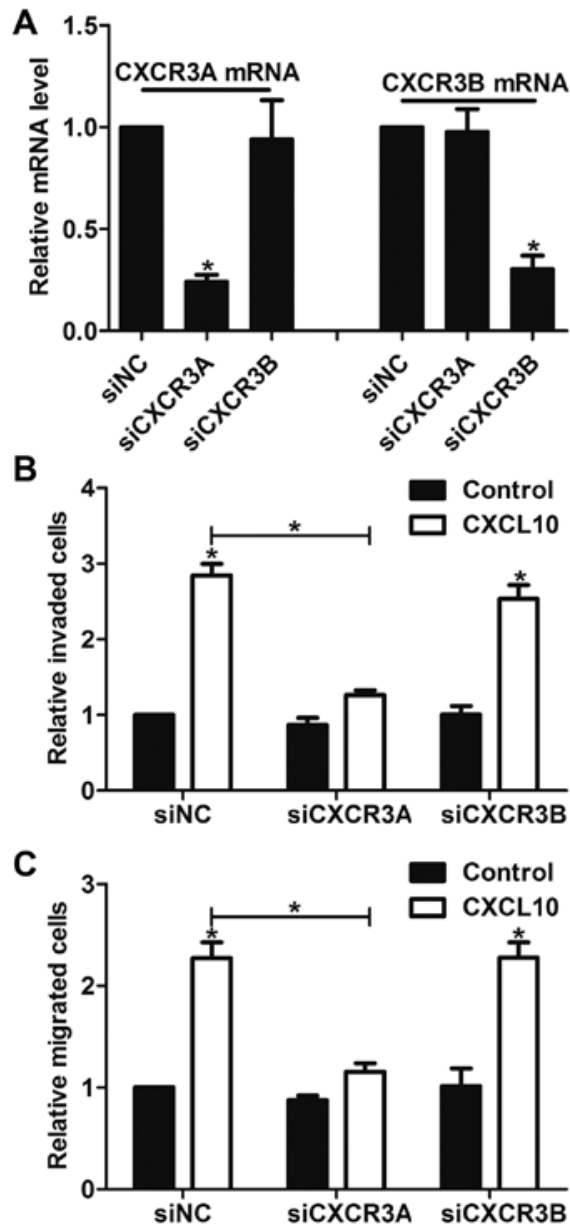

Figure 3. CXCL10 induces gastric cancer cell invasion and migration through CXCR3A in vitro. (A) Knockdown efficiency of CXCR3A and CXCR3B was detected by real-time PCR. (B) In vitro invasion and (C) migration assay in siNC, CXCR3A siRNA (siCXCR3A) and CXCR3B siRNA (siCXCR3B) cells treated with or without CXCL10. ${ }^{*} \mathrm{P}<0.05$.

greatly inhibited (Fig. 7C), as compared to tumor tissues of shNC group.

\section{Discussion}

Chemokines are well known to modulate tumor progression via activation of chemokine receptors in the membrane of tumor cells (13). Belonging to the CXC chemokine receptor subfamily, CXCR3 has three variants in human cells (CXCR3A, CXCR3B and CXCR3alt) (14). Until now little is known about the role of CXCR3 in gastric cancer. In our study, we found that CXCR3A expression was increased, while CXCR3B expression was decreased in gastric cancer tissues and cells. We also demonstrated that CXCR3A participated in the growth, migration, invasion and metastasis of gastric cancer cells in vitro and in vivo, supporting the notion that CXCR3A acts as a positive mediator in gastric cancer progression.

The level of CXCR3 has been found to be elevated in many cancer tissues and cells $(15,16)$. However, the three mRNA splice variants of CXCR3 (CXCR3A, CXCR3B and CXCR3alt) may show different level in the same tumor. Furuya et al found that the mRNA levels of CXCR3A and CXCR3alt are upregulated, while the mRNA expression of 

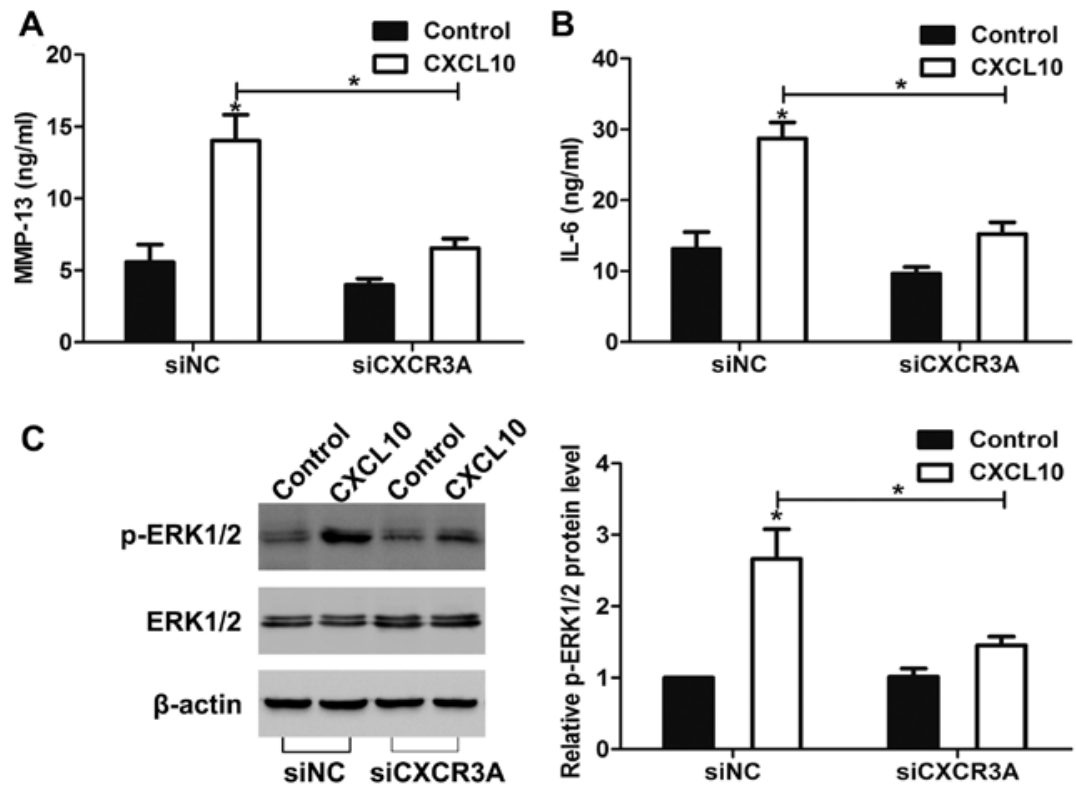

Figure 4. Effects of CXCR3A on the secretion of matrix metalloproteinase (MMP)-13 and IL-6 and activation of ERK1/2. Secretion of (A) MMP-13 and (B) IL-6 in siNC and CXCR3A siRNA (siCXCR3A) cells treated with or without CXCL10 was detected by ELISA assay. (C) Activation of ERK1/2 in siNC and siCXCR3A cells treated with or without CXCL10 was detected by western blot analysis. ${ }^{*} \mathrm{P}<0.05$.
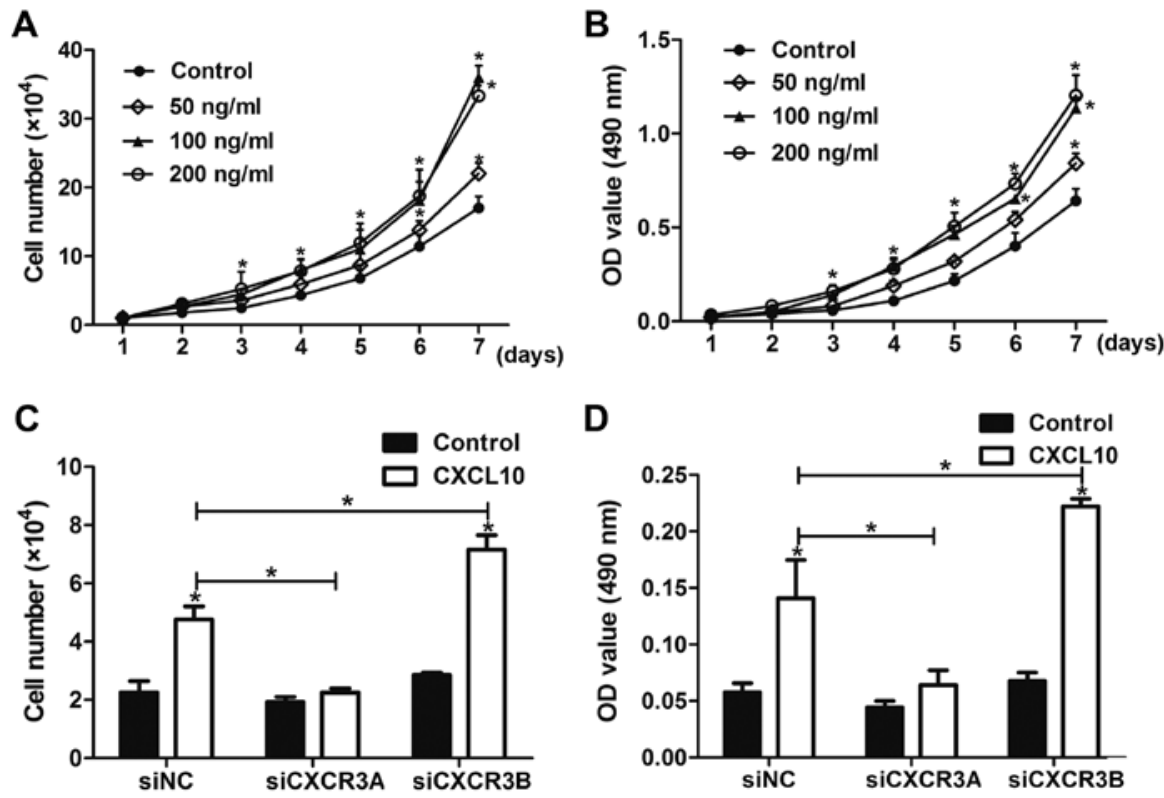

Figure 5. Effect of CXCR3A on CXCL10-mediated in vitro growth. Cells were stimulated with 50, 100 and $200 \mathrm{ng} / \mathrm{ml}$ CXCL10, respectively, every day for 7 days. In vitro growth was determined by (A) cell count assay and (B) CCK-8 assay. After knockdown of CXCR3A, cells were stimulated with $100 \mathrm{ng} / \mathrm{ml}$ CXCL10 for $72 \mathrm{~h}$, in vitro growth was determined by (C) cell count assay and (D) CCK-8 assay. ${ }^{*} \mathrm{P}<0.05$.

CXCR3B is downregulated in clear cell ovarian cancer (9). Here, we found that the mRNA expression of CXCR3A was upregulated in gastric cancer tissues and cells, but the level of CXCR3B was downregulated compared with normal control (corresponding gastric tissues or GES-1 cells). Moreover, CXCR3alt expression was not altered. The results suggest that these splice variants may play different role in gastric cancer.

It is reported that CXCR3 signaling promotes the growth of liver tumor (17). Further studies found that CXCR3B expression correlates with tumor necrosis and can mediate growth-inhibitory signals in human renal cancer cells $(18,19)$, whereas downregulation of CXCR3A inhibits prostate cancer PC-3 cell proliferation (10). However, the role of CXCR3 in gastric cancer growth remains unclear. In our study, we found that knockdown of CXCR3A suppressed the growth of gastric cancer cells, whereas knockdown of CXCR3B promoted gastric cancer growth, confirming the conclusion that CXCR3A and CXCR3B display converse roles in regulating gastric cancer cell growth.

$\mathrm{Pu}$ et al found that high expression of CXCR3 is an independent prognostic factor in glioblastoma patients that promotes an invasive phenotype (20). However, Wu et al reported that 

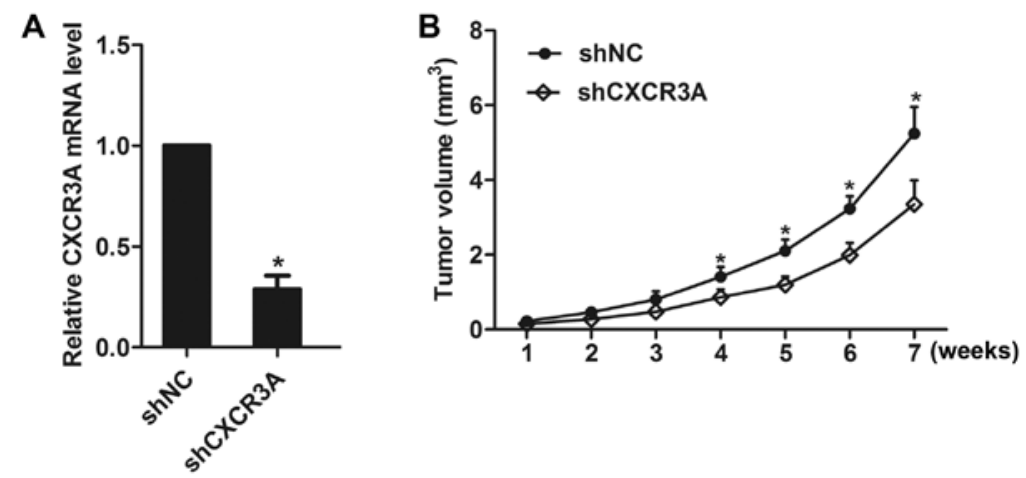

C
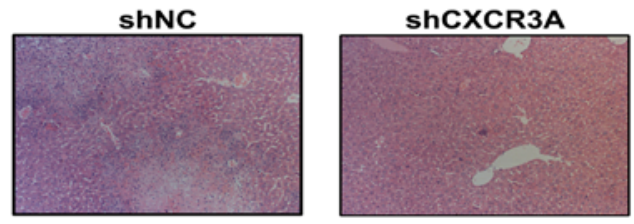

D

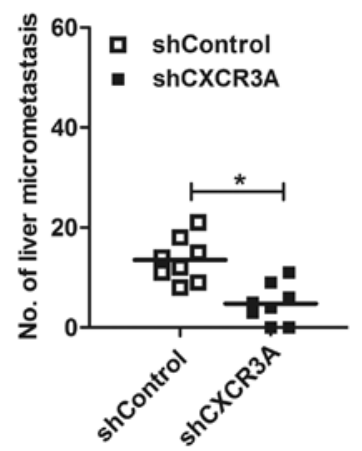

Figure 6. Effects of CXCR3A on gastric cancer cell growth and metastasis in vivo. (A) Stable knockdown of CXCR3A in AGS cells was detected by real-time PCR. (B) Average tumor volume was measured and calculated in shNC and CXCR3A shRNA (shCXCR3A) group. (C) Micrometastasis in the liver section was observed under a microscope (magnification, x200). (D) Number of micrometastasis in the liver of shNC and shCXCR3A group was counted. "P<0.05.
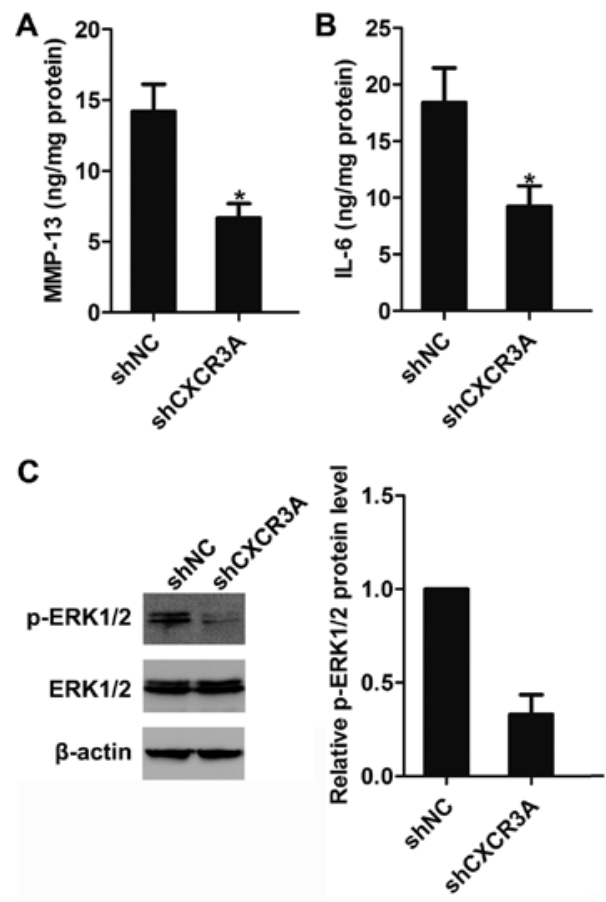

Figure 7. Knockdown of CXCR3A affected matrix metalloproteinase (MMP)-13 and IL-6 expression and ERK1/2 activation. Expression of (A) MMP-13, (B) IL-6 and in the tumor tissues of shNC and CXCR3A shRNA (shCXCR3A) group. (C) Activation of ERK1/2 in the tumor tissues of shNC and shCXCR3A group. ${ }^{*} \mathrm{P}<0.05$. overexpression of CXCR3B in prostate cancer DU-145 cells decreases cell invasion (11). The function of CXCR3A and CXCR3B in gastric cancer cell invasion is elusive. Here, we found that activation of CXCR3 by CXCL10 stimulated the invasion and migration of gastric cancer cells. Further, knockdown of CXCR3A inhibited the CXCL10-mediated cell invasion and migration, but knockdown of CXCR3B did not affect the invasion and migration of gastric cancer cells. Studies have reported that CXCR3 can promote the metastasis of breast and osteosarcoma metastasis $(21,22)$. In renal cell carcinoma, CXCR3 and CXCR3A expression is found to be significantly higher in metastatic than in non-metastatic carcinoma samples (23). In our study, we found that knockdown of CXCR3A suppressed the metastasis of gastric cancer cells in vivo.

The MMP family plays a critical role in tumor progression through ECM turnover and cancer cell migration. Shen and Cao have reported that overexpression of CXCR3 increases the expressions of MMP-1 and -3 in prostate cancer cells (10). Here, we found that CXCL10 increased the expression of MMP-13 in gastric cancer cells, whereas knockdown of CXCR3A decreased the MMP-13 expression in vitro. Interleukins are a group of secreted proteins and signaling molecules, and are involved in cancer progression $(24,25)$. As a member of interleukin family, IL-6 is reported to be required for cancer invasion and migration $(26,27)$. Jenkins et al reported 
that CXCR3 signaling increases IL-8 expression in melanoma (28), but the effect of CXCR3A on IL-6 expression in gastric cancer cells is unclear. Here, we found that knockdown of CXCR3A downregulated the CXCL10-mediated secretion of IL-6 in vitro. Moreover, knockdown of CXCR3A inhibited the expression of MMP-13 and IL-6 in vivo. ERK1/2 pathway plays an important role in regulating tumorigenicity and tumor development (29). In this study, we found that CXCR3A could induce the activation of ERK1/2 in vitro and in vivo.

In conclusion, our study demonstrates that CXCR3A is overexpressed in gastric cancer tissues and cells. CXCL10 stimulation promotes the growth of gastric cancer cells via CXCR3A. Moreover, CXCR3A contributes to the invasion and metastasis of gastric cancer cells in vitro and in vivo, probably via regulating MMP-13 and IL-6 expression and ERK1/2 activation. Thus, CXCR3A could be a biomarker for gastric cancer diagnosis and treatment.

\section{References}

1. Torre LA, Bray F, Siegel RL, Ferlay J, Lortet-Tieulent J and Jemal A: Global cancer statistics, 2012. CA Cancer J Clin 65 87-108, 2015.

2. De Vita F, Di Martino N, Fabozzi A, Laterza MM, Ventriglia J, Savastano B, Petrillo A, Gambardella V, Sforza V, Marano L, et al Clinical management of advanced gastric cancer: The role of new molecular drugs. World J Gastroenterol 20: 14537-14558, 2014.

3. Singh UP, Venkataraman C, Singh R and Lillard JW Jr: CXCR3 axis: Role in inflammatory bowel disease and its therapeutic implication. Endocr Metab Immune Disord Drug Targets 7: 111-123, 2007.

4. Lacotte S, Brun S, Muller S and Dumortier H: CXCR3, inflammation, and autoimmune diseases. Ann N Y Acad Sci 1173: 310-317, 2009.

5. Billottet C, Quemener C and Bikfalvi A: CXCR3, a double-edged sword in tumor progression and angiogenesis. Biochim Biophys Acta 1836: 287-295, 2013

6. Lo BK, Yu M, Zloty D, Cowan B, Shapiro J and McElwee KJ: CXCR3/ligands are significantly involved in the tumorigenesis of basal cell carcinomas. Am J Pathol 176: 2435-2446, 2010.

7. Wu Z, Han X, Yan J, Pan Y, Gong J, Di J, Cheng Z, Jin Z, Wang Z, Zheng $\mathrm{Q}$, et al: The prognostic significance of chemokine receptor CXCR3 expression in colorectal carcinoma. Biomed Pharmacother 66: 373-377, 2012.

8. Ma B, Khazali A and Wells A: CXCR3 in carcinoma progression. Histol Histopathol 30: 781-792, 2015.

9. Furuya M, Yoneyama T, Miyagi E, Tanaka R, Nagahama K, Miyagi Y, Nagashima Y, Hirahara F, Inayama Y and Aoki I: Differential expression patterns of CXCR3 variants and corresponding CXC chemokines in clear cell ovarian cancers and endometriosis. Gynecol Oncol 122: 648-655, 2011.

10. Shen D and Cao X: Potential role of CXCR3 in proliferation and invasion of prostate cancer cells. Int J Clin Exp Pathol 8: 8091-8098, 2015.

11. Wu Q, Dhir R and Wells A: Altered CXCR3 isoform expression regulates prostate cancer cell migration and invasion. Mol Cancer 11: 3, 2012.

12. Hu M, Li K, Maskey N, Xu Z, Yu F, Peng C, Li Y and Yang G: Overexpression of the chemokine receptor CXCR3 and its correlation with favorable prognosis in gastric cancer. Hum Pathol 46: $1872-1880,2015$
13. Salazar N, Castellan M, Shirodkar SS and Lokeshwar BL: Chemokines and chemokine receptors as promoters of prostate cancer growth and progression. Crit Rev Eukaryot Gene Expr 23: 77-91, 2013

14. Van Raemdonck K, Van den Steen PE, Liekens S, Van Damme J and Struyf S: CXCR3 ligands in disease and therapy. Cytokine Growth Factor Rev 26: 311-327, 2015.

15. Murakami T, Kawada K, Iwamoto M, Akagami M, Hida K, Nakanishi Y, Kanda K, Kawada M, Seno H, Taketo MM, et al: The role of CXCR3 and CXCR4 in colorectal cancer metastasis. Int J Cancer 132: 276-287, 2013.

16. Goldberg-Bittman L, Neumark E, Sagi-Assif O, Azenshtein E, Meshel T, Witz IP and Ben-Baruch A: The expression of the chemokine receptor CXCR3 and its ligand, CXCL10, in human breast adenocarcinoma cell lines. Immunol Lett 92: 171-178, 2004.

17. Ling CC, Ng KT, Shao Y, Geng W, Xiao JW, Liu H, Li CX, Liu XB, Ma YY, Yeung WH, et al: Post-transplant endothelial progenitor cell mobilization via CXCL10/CXCR3 signaling promotes liver tumor growth. J Hepatol 60: 103-109, 2014

18. Datta D, Banerjee P, Gasser M, Waaga-Gasser AM and Pal S: CXCR3-B can mediate growth-inhibitory signals in human renal cancer cells by down-regulating the expression of heme oxygenase-1. J Biol Chem 285: 36842-36848, 2010.

19. Gacci M, Serni S, Lapini A, Vittori G, Alessandrini M, Nesi G, Palli D and Carini M: CXCR3-B expression correlates with tumor necrosis extension in renal cell carcinoma. J Urol 181: 843-848, 2009.

20. Pu Y, Li S, Zhang C, Bao Z, Yang Z and Sun L: High expression of CXCR3 is an independent prognostic factor in glioblastoma patients that promotes an invasive phenotype. J Neurooncol 122: 43-51, 2015.

21. Ma X, Norsworthy K, Kundu N, Rodgers WH, Gimotty PA, Goloubeva O, Lipsky M, Li Y, Holt D and Fulton A: CXCR3 expression is associated with poor survival in breast cancer and promotes metastasis in a murine model. Mol Cancer Ther 8: 490-498, 2009.

22. Pradelli E, Karimdjee-Soilihi B, Michiels JF, Ricci JE, Millet MA, Vandenbos F, Sullivan TJ, Collins TL, Johnson MG, Medina JC, et al: Antagonism of chemokine receptor CXCR3 inhibits osteosarcoma metastasis to lungs. Int J Cancer 125: 2586-2594, 2009.

23. Utsumi T, Suyama T, Imamura Y, Fuse M, Sakamoto S, Nihei N, Ueda T, Suzuki H, Seki N and Ichikawa T: The association of CXCR3 and renal cell carcinoma metastasis. J Urol 192: 567-574, 2014.

24. Anestakis D, Petanidis S, Kalyvas S, Nday CM, Tsave O, Kioseoglou E and Salifoglou A: Mechanisms and applications of interleukins in cancer immunotherapy. Int J Mol Sci 16: 1691-1710, 2015.

25. Lippitz BE: Cytokine patterns in patients with cancer: A systematic review. Lancet Oncol 14: e218-e228, 2013.

26. Wang G, Ye Y, Zhang X and Song J: Bradykinin stimulates IL-6 production and cell invasion in colorectal cancer cells. Oncol Rep 32: 1709-1714, 2014.

27. Dehai C, Bo P, Qiang T, Lihua S, Fang L, Shi J, Jingyan C, Yan Y, Guangbin W and Zhenjun Y: Enhanced invasion of lung adenocarcinoma cells after co-culture with THP-1-derived macrophages via the induction of EMT by IL-6. Immunol Lett 160: 1-10, 2014

28. Jenkins $M H$, Brinckerhoff $\mathrm{CE}$ and Mullins DW: CXCR3 signaling in BRAFWT melanoma increases IL-8 expression and tumorigenicity. PLoS One 10: e0121140, 2015.

29. Burotto M, Chiou VL, Lee JM and Kohn EC: The MAPK pathway across different malignancies: A new perspective. Cancer 120: 3446-3456, 2014. 